Original Paper http://ajol.info/index.php/ijbcs http://indexmedicus.afro.who.int

\title{
Déterminants socio-économiques de l'adoption des pratiques agro-écologiques en production maraîchère dans la vallée du Niger au Bénin
}

\author{
Claude Codjo KPADENOU ${ }^{1 *}$, Clarisse TAMA ${ }^{2}$, Baké DADO TOSSOU ${ }^{3}$ et \\ Jacob Afouda YABI ${ }^{1}$ \\ ${ }^{1}$ Laboratoire d'Analyse et de Recherches sur les Dynamiques Economiques et Sociales (LARDES), Faculté \\ d'Agronomie (FA), Université de Parakou (UP), BP 123, République du Bénin. \\ ${ }^{2}$ Département de Sociologie, Faculté des Lettres, Arts et Sciences Humaines (FLASH), Faculté d'Agronomie \\ (FA), Université de Parakou (UP), BP 123, République du Bénin. \\ ${ }^{3}$ Laboratoire Société-Environnement (laSEn), Université de Parakou (UP), BP 27, République du Bénin. \\ *Auteur correspondant; E-mail: kpadenouclaude@gmail.com, Tél. : (+229) 97326917
}

\section{RESUME}

Cette étude tente d'identifier les facteurs qui favorisent ou limitent l'adoption des pratiques agro-écologiques dans le secteur maraîcher dans la vallée du Niger au Bénin. Pour atteindre cet objectif, des entretiens individuels sur la base d'un questionnaire semi-structuré ont été conduits auprès de 280 maraîchers, chefs de ménages, choisis de manière aléatoire dans sept villages de ladite vallée. Des données relatives aux caractéristiques socioéconomiques des maraîchers et aux pratiques agro-écologiques utilisées dans la production maraîchère ont été collectées. Le modèle de régression Logit a été utilisé. Les résultats révèlent que le sexe du maraîcher, le nombre d'actifs agricoles et le niveau d'instruction et le contact ont une influence positive sur l'adoption des pratiques biologique, mécanique et recours à d'autres alternatives aux pesticides; par contre, l'expérience dans le maraîchage et la superficie emblavée en maraîchage influencent négativement cette pratique. Quant à l'adoption des semences améliorées, l'expérience dans le maraîchage, la superficie et le nombre d'actifs agricoles l'influence positivement. La pratique d'agroforesterie quant à elle est déterminée positivement par le nombre d'actifs agricoles et le mode d'accès à la terre, mais négativement déterminée par l'expérience. Pour les pratiques de conservation des sols et de l'eau, l'expérience et la superficie influencent négativement leur adoption tandis que le nombre d'actifs agricoles, le niveau d'instruction et le statut foncier déterminent positivement l'adoption de ces pratiques. La pratique traditionnelle qu'est la rotation et l'association de cultures appropriées est déterminée positivement par l'expérience et négativement par le nombre d'actifs agricoles. Enfin, le nombre d'actifs agricoles et le sexe influencent négativement l'adoption de la pratique de l'association des cultures et l'élevage contrairement au nombre d'années d'expérience dans le maraîchage. Au vu de ces résultats, la promotion des pratiques agro-écologiques en vue d'augmenter leur niveau d'adoption, passe indubitablement par un suivi rapproché et une formation des maraîchers.

() 2019 International Formulae Group. All rights reserved

Mots clés: Maraîchage, pratiques agro-écologiques, régression logistique, vallée du Niger, Nord-Bénin. 


\title{
Socio-economic determinants of the adoption of agro-ecological practices in market production in the Niger valley in Benin
}

\begin{abstract}
This study tries to identify the factors of the adoption of agro-ecological practices in the market gardening sector in the Niger valley in Benin. Thus, a survey was conducted among 280 market gardeners sampled in seven villages in the said valley at random. Based on an individual questionnaire, data on the socioeconomic characteristics of market gardeners and agro-ecological practices used in vegetable production were collected. The Logit regression model was used. The results reveal that the number of assets, the level of education and the contact with an NGO or project have a positive influence on the adoption of biological, mechanical practices and other alternatives to pesticides; on the other hand, sex, experience in market gardening and the area sown in market gardening negatively influence this practice. As for the adoption of improved seeds, the experience variables, the area, the number of agricultural workers, the level of education and membership in a group influence it positively. The agroforestry practice is positively determined by the number of agricultural workers and the mode of access to land, but negatively by the experience and membership of a group. For soil and water conservation practices; experience and area negatively influence their adoption, while the number of agricultural assets and land status positively determine the adoption of this practice. The traditional practice of rotation and the association of appropriate cultures is positively determined by experience; negatively by the number of agricultural assets and the level of education. Lastly, the number of agricultural assets and gender negatively influence the adoption of the practice of the combination of crops and livestock, contrary to the number of years of experience in market gardening. In view of these results, the promotion of agro-ecological practices in order to increase their level of adoption, undoubtedly passes by a close monitoring and training of market gardeners.
\end{abstract}

(C) 2019 International Formulae Group. All rights reserved

Keywords: Market gardening, agro-ecological practicies, logic regression, North Benin, Niger valley

\section{INTRODUCTION}

Au Bénin, la lutte contre la pauvreté et l'insécurité alimentaire particulièrement dans le monde rural constitue une préoccupation majeure des acteurs de développement. Cette lutte est rendue difficile dans le monde rural par une faible productivité agricole (Seye et al., 2017). L'une des voies pour améliorer les conditions de vie des producteurs et lutter contre la pauvreté est la diversification des filières agricoles. Elle permet de réduire les risques financiers et d'améliorer les revenus des producteurs (Ahouandjinou et al., 2010). Une de ces filières agricoles est le maraîchage, cible de cette étude. Il figure aujourd'hui parmi les douze filières prioritaires identifiées et retenues par le Gouvernement béninois (PSDSA, 2017).

L'agriculture maraîchère peut cependant offrir plusieurs bénéfices importants pour les ménages qui s'y adonnent (Biaou et al., 2016). Elle participe d'une part à l'approvisionnement en aliments frais localement produits pour les citoyens urbains de diverses classes sociales. Elle a des impacts positifs sur la sécurité alimentaire et nutritionnelle ainsi que sur les conditions économiques des ménages à faible revenu. La proximité des marchés et des services urbains offre aussi des avantages intéressants pour les producteurs qui peuvent écouler leur marchandise hautement périssable sur les marchés urbains et s'approvisionner en intrants dans les commerces et les industries urbaines (Gravel, 2016).

Cependant, le maraîchage présente des risques pour la santé et la qualité de l'environnement puisqu'il utilise parfois de grandes quantités d'intrants minéraux ou des sources de produits phytosanitaires inappropriés pour la culture des fruits et légumes. Les dernières décennies de domination d'agriculture conventionnelle nous ont permis d'apprendre que les pratiques 
tels que l'usage abondant d'intrants chimiques et synthétiques, le lourd travail des sols, l'utilisation d'une faible diversité de cultivars à haut rendement et les techniques d'irrigation inadaptée aux conditions locales engendrent des impacts négatifs importants sur le plan environnemental et écologique (Trabelsi, 2017). Aujourd'hui, de nombreuses solutions de rechange sont proposées pour remplacer le modèle agricole conventionnel mondial. L'agroécologie fait partie de ces solutions de rechange et propose une vision plus intégrative de l'agriculture. Elle propose une vision des systèmes alimentaires en entier plutôt que des paramètres agricoles qui permettent d'augmenter la production alimentaire.

En effet, une étude exploratoire du Projet de Soutien à l'Extension de l'Agriculture Agro-Ecologique au Bénin (2011) a permis d'identifier six grands groupes de pratiques agro-écologiques. Il s'agit de : la diversification et la rotation des cultures, l'agroforesterie, l'association des cultures et l'élevage, la gestion et la conservation des sols et l'eau, la lutte biologique et mécanique et le recours à d'autres alternatives aux pesticides et l'utilisation des semences paysannes et améliorées. Toutefois, ces innovations sont peu adoptées par les maraîchers. Il apparaît donc opportun de chercher à comprendre quels sont les facteurs qui limitent ou favorisent l'adoption de ces pratiques afin d'œuvrer efficacement pour l'acceptation de ses pratiques en milieu rural en vue d'une meilleure gestion de la fertilité des sols et des ravageurs. C'est dans cette perspective que s'inscrit la présente étude. Elle vise spécifiquement à identifier les variables susceptibles d'influencer la probabilité de l'adoption des différentes pratiques agroécologiques en production maraîchère dans la vallée du Niger au Bénin.

\section{MATERIELS ET METHODES Matériels \\ Zone d'étude}

La Vallée du Niger (côté Bénin) est située à l'extrême Nord de la République du
Bénin. Elle s'étale sur les Communes de Malanville et de Karimama se trouvant entre $11^{\circ} 30$ et $12^{\circ} 30$ de latitude Nord d'une part et les méridiens $2^{\circ} 43$ et $3^{\circ} 20$ de longitude Est d'autre part. Ces communes sont limitées au Nord par le fleuve Niger, au Sud par les Communes de Banikoara, Kandi et Ségbanan, à l'Est par le fleuve Niger et la République Fédérale du Nigeria et à l'Ouest par le Burkina Faso.

Les villages étudiés par commune ont été choisis selon leur accessibilité en toute saison et leurs fortes productions en cultures maraîchères. Il s'agit de: Toumboutou, Garou-Tédji, Madécali et Monkassa (dans la commune de Malanville), Kargui, Birni-Lafia et Kotchi (dans la commune de Karimama). Cette inégale répartition est due au fait que la commune de Malanville constitue la grande zone de production maraîchère dans la Vallée du Niger.

\section{Echantillonnage et collecte des données}

Les unités de recherche sont des maraîchers, chefs de ménage de sept villages à forte production de cultures maraîchères dans la zone d'étude. La taille minimale de l'échantillon a été obtenue en utilisant l'approximation normale de la distribution binomiale proposée par (Dagnelie, 1998) : N $=\left[\left(\mathrm{U}_{1-\alpha / 2}\right)^{2} \times \mathrm{p}(1-\mathrm{p})\right] / \mathrm{d}^{2}$, avec $\mathrm{U}_{1-\alpha / 2}$ la valeur de la variable aléatoire normale pour la valeur de probabilité de $1-\alpha / 2, \alpha$ étant le risque d'erreur. Pour $\alpha=5 \%$, la probabilité de $1-\alpha / 2$ est égale à 0,975 et on a $U_{1-\alpha / 2}=1,96$. p est la proportion de personnes qui s'adonnent à la production de cultures de contre-saison dans le milieu d'étude et d la marge d'erreur d'estimation, retenue à $5 \%$ dans cette étude.

A partir de la valeur de $\mathrm{p}=0,24$, proportion de la population qui s'adonne au maraichage dans la zone d'étude issues des résultats de la phase exploratoire de l'étude, deux cent quatre-vingts (280) maraîchers ont été sélectionnés au total, soit quarante (40) enquêtés par village. Dans chaque village, les personnes enquêtées ont été identifiées selon un échantillonnage aléatoire simple. De plus, des focus groups ont été réalisés dans chaque village. Ces focus groups ont été réalisés avec les maraîchers, les agents d'encadrement et les 
agents des ONG qui interviennent dans le domaine. Au cours de ces focus groups, les maraîchers se sont prononcés sur les différentes pratiques agro-écologiques retenus. Il s'agit de: la diversification et la rotation des cultures, l'agroforesterie, l'association des cultures et l'élevage, la gestion et la conservation des sols et l'eau, la lutte biologique et mécanique et recours à d'autres alternatives aux pesticides et l'utilisation des semences paysannes et améliorées. Enfin, des entretiens ont été également faits sur la base d'un questionnaire semi-structuré pour collecter des données primaires. Ces données sont: le sexe, l'expérience dans le maraîchage, la superficie emblavée en maraîchage, le nombre d'actifs, le niveau d'instruction, l'accès à la vulgarisation, le contact avec une ONG ou un projet, l'appartenance à un groupement, l'accès au crédit et le mode d'accès à la terre.

\section{Approche méthodologique \\ Cadre théorique de l'adoption de technologies}

Le producteur fonde sa décision d'adoption des technologies sur la base du principe de rationalité notamment l'hypothèse de maximisation de l'utilité de la théorie néoclassique. Il n'adopte ou n'intensifie la technologie que si la profitabilité anticipée est supérieure à celle de la non adoption ou non intensification (Marenya et al., 2007). Le producteur rationnel préfère la pratique agricole qui lui procure le plus d'utilité. Ainsi, il est donc possible d'émettre l'hypothèse selon laquelle «la probabilité d'adoption des pratiques agro-écologiques est fonction de facteurs économiques liés au maraîcher ». Les rapports sociaux influençant les choix opérés par le producteur (CIRADGRET, 2002), l'hypothèse précédente apparait incomplète. Toutefois, il est bien établi que la raison pour laquelle les agriculteurs adoptent une technologie va au-delà de ces considérations de la théorie néoclassique. Tenant donc compte de l'environnement de l'exploitation agricole (Relations avec d'autres agents économiques: agriculteurs voisins, propriétaires fonciers, commerçants, agents de développement rural, etc.), la probabilité d'adoption des pratiques agroécologiques est fonction de facteurs sociodémographiques et économiques qui leur sont liés. En nous basant sur les fondements théoriques ci-dessus et des résultats de plusieurs études antérieures notamment ceux de Diogo al. (2017) et de Yabi et al. (2016), il est possible d'identifier les facteurs affectant la probabilité d'adoption des pratiques agroécologiques comme une fonction de certaines variables exogènes sociodémographiques et économiques. Pour analyser l'adoption des pratiques agro-écologiques, la présente étude utilise le modèle Logit qui a montré ses preuves dans des études similaires notamment celles de Issoufou et al. (2017), Yabi et al. (2016) et Roussy et al. (2015).

La décision d'adopter une innovation n'intervient que lorsque l'effet combiné des facteurs atteint une valeur à partir de laquelle le décideur accepte d'utiliser ou adopter l'innovation. En se mettant dans l'hypothèse que l'effet est mesuré par un indice non observable $\mathrm{I}_{\mathrm{d}}$ pour le décideur $\mathrm{d}$ et $\mathrm{I}_{0 \mathrm{~d}}$ la valeur critique de l'indice à partir de laquelle il adopte la technologie, deux cas de figures peuvent se présenter :

-Si $\mathrm{I}_{\mathrm{d}}$ est supérieur ou égal à $\mathrm{I}_{0 \mathrm{~d}}$, alors il adopte la technologie et la variable d'adoption $\mathrm{Y}$ prend la valeur 1. Plus l'indice $I_{d}$ est supérieur à la valeur critique, plus la probabilité pour que le producteur adopte est élevée. Si $\mathrm{I}_{\mathrm{d}}$ est inférieur à $\mathrm{I}_{0 \mathrm{~d}}$, il rejette l'innovation et $\mathrm{Y}$ est égal à 0 .

En formulation mathématique, il vient que :

$$
\left\{\begin{array}{l}
I_{d} \geq I_{0 d} \Rightarrow \mathrm{Y}=1 \\
I_{d}<I_{0 d} \Rightarrow \mathrm{Y}=0
\end{array}\right.
$$

-Pour l'individu $d$, l'indice $I_{d}$ peut être une combinaison linéaire de variables $\mathrm{Xi}$ qui déterminent l'adoption et de coefficients $\beta \mathrm{i}$ à estimer. Son expression est alors mathématiquement donnée par :

$I_{d}=\sum_{i=1}^{k} \beta_{i} X_{i d}$

Avec $X_{\text {id }}$ la iième variable indépendante expliquant l'adoption de la technologie par l'individu $\mathrm{d}$ et $\beta \mathrm{i}$ son paramètre correspondant à estimer.

La probabilité $\mathrm{P}_{\mathrm{d}}$ pour que l'individu d adopte l'innovation est alors : 
$\mathrm{Pd}=\mathrm{P}(\mathrm{Y}=1)$

Comme l'indice $\mathrm{I}_{0 \mathrm{~d}}$ est une variable aléatoire, si nous désignons par $\mathrm{F}$ sa fonction de probabilité cumulée ou fonction de répartition, il vient que :

$\left\{P(Y=1)=P\left(I_{0 d} \leq I_{d}=F\left(I_{d}\right)\right.\right.$

$\left\{P(Y=0)=P\left(1-F\left(I_{d}\right)\right.\right.$

La forme fonctionnelle de $\mathrm{F}$ est déterminée par celle de la fonction de densité de probabilité de la variable aléatoire Id. Pour le modèle Logit, il s'agit d'une fonction logistique de la forme :

$F(x)=\frac{1}{1+e^{-X}}=\frac{1}{1+e^{-\left(\beta_{0}+\beta_{i} Z\right)}}$

\section{Modèle empirique}

Dans le cadre de la présente étude, les variables dépendantes des modèles Logit sont l'adoption des pratiques agro-écologiques identifiées : la diversification et la rotation des cultures, l'agroforesterie, l'association des cultures et l'élevage, la gestion et la conservation des sols et l'eau, la lutte biologique et mécanique et le recours à d'autres alternatives aux pesticides et l'utilisation des semences paysannes et améliorées. A partir de la littérature théorique et économétrique sur l'adoption des technologies des travaux de CYMMIT (1993); Houndekon et al. (1996); Sale et al. (2014) et Yabi et al. (2016) et des données d'enquête, les variables explicatives susceptibles d'influencées l'adoption de ces pratiques agro-écologiques sont résumées dans le Tableau 1.
L'équation empirique issue $\mathrm{du}$ modèle théorique, se présente comme suit :

$$
P\left(Y_{i}=1 / A D O P\right)=\frac{1}{1+e^{-X}}
$$

Avec $\quad X=\beta_{0}+\beta_{1} M A L A N+\beta_{2} S E X+$ $\beta_{3}$ ACTPRIN $+\beta_{4} E X P+\beta_{5} S U P+$ $\beta_{6} A C T I F+\beta_{7} E D U C F+\beta_{8} E D U C I+$ $\beta_{9}$ ACTPRIN $+\beta_{10} M F V D+\beta_{11}$ GROUP + $\beta_{12} O N G+\beta_{13} V U L G+\beta_{14} C R E D+e_{1}$

(7)

Où $\beta_{0}$ est le terme constant ; $\beta_{i}$ les coefficients à estimer, et ei les termes d'erreur. Plusieurs techniques sont utilisées pour juger de la qualité du modèle : elle peut être donnée par la vraisemblance du modèle qui suit une loi de Chi-deux. Le modèle est dit globalement bon, lorsque la valeur de la vraisemblance est supérieure à celle du Chi deux au même degré de liberté à un seuil donné $(1 \%, 5 \%$ ou $10 \%)$, ou directement lorsque la probabilité de LR est inférieure au seuil de signification choisi. Les signes indiquent dans quel sens la variation de la variable explicative influe sur la variation de la variable expliquée. A chaque signe des coefficients, est associée une signification qui revêt une grande importance. Leur signification est donnée par une probabilité qui indique dans quel intervalle de confiance on peut compter sur le signe. Les logiciels SPSS et STATA ont été utilisés respectivement pour les statistiques descriptives et les régressions économétriques.

Tableau 1 : Description et statistiques descriptives des variables incluses dans les modèles Logit.

\begin{tabular}{|c|c|c|c|c|c|c|}
\hline \multirow[t]{2}{*}{ Variables } & \multirow[t]{2}{*}{ Code } & \multicolumn{2}{|c|}{$\begin{array}{l}\text { Variables } \\
\text { quantitatives }\end{array}$} & \multicolumn{2}{|c|}{ Variables qualitatives } & \multirow[t]{2}{*}{$\begin{array}{l}\text { Signes } \\
\text { attendus }\end{array}$} \\
\hline & & Moyenne & $\begin{array}{l}\text { Ecart } \\
\text {-type }\end{array}$ & Modalité & Fréquence & \\
\hline Superficie cultivée (ha) & SUP & 2,08 & 1,65 & & & - \\
\hline Expérience (années) & EXP & 17,25 & 7,48 & & & + \\
\hline Actif agricole & ACTI & 5,08 & 2,87 & & & + \\
\hline Commune & $\mathrm{COM}$ & & & $\begin{array}{l}1=\text { Malanville } \\
0=\text { Karaimama }\end{array}$ & $\begin{array}{l}160(54,14) \\
120(42,86)\end{array}$ & \pm \\
\hline Sexe & SEXE & & & $\begin{array}{l}1=\text { Homme } \\
0=\text { Femme }\end{array}$ & $\begin{array}{l}259(92,5) \\
21(7,5)\end{array}$ & \pm \\
\hline
\end{tabular}




\begin{tabular}{|c|c|c|c|c|}
\hline $\begin{array}{l}\text { Maraîchage comme } \\
\text { activité principale }\end{array}$ & $\begin{array}{l}\text { ACTPR } \\
\text { IN }\end{array}$ & $\begin{array}{l}1=\text { Oui } \\
0=\text { Non }\end{array}$ & $\begin{array}{l}72(25,71) \\
208(74,29)\end{array}$ & + \\
\hline Mode d'accès à la terre & MFVD & $\begin{array}{l}\text { 1=Accès direct à } \\
\text { la terre } \\
0=\text { accès indirect } \\
\text { à la terre }\end{array}$ & $\begin{array}{l}213(75,90) \\
67(45,86)\end{array}$ & + \\
\hline Education formelle & $\begin{array}{l}\text { EDUC } \\
F\end{array}$ & $\begin{array}{l}1=\text { Oui } \\
0=\text { Non }\end{array}$ & $\begin{array}{l}92(32,86) \\
188(67,14)\end{array}$ & + \\
\hline Education informelle & $\begin{array}{l}\text { EDUCI } \\
\mathrm{N}\end{array}$ & $\begin{array}{l}1=\text { Oui } \\
0=\text { Non }\end{array}$ & $\begin{array}{l}44(15,71) \\
236(84,29)\end{array}$ & + \\
\hline Accès à la vulgarisation & VULG & $\begin{array}{l}1=\text { Oui } \\
0=\text { Non }\end{array}$ & $\begin{array}{l}220(78,57) \\
60(21,43)\end{array}$ & + \\
\hline $\begin{array}{l}\text { Contacts avec ONG ou } \\
\text { projet }\end{array}$ & ONG & $\begin{array}{l}1=\text { Oui } \\
0=\text { Non }\end{array}$ & $\begin{array}{l}214(76,43) \\
66(23,57)\end{array}$ & + \\
\hline $\begin{array}{l}\text { Appartenance à un } \\
\text { groupe }\end{array}$ & $\begin{array}{l}\text { GROU } \\
\mathrm{P}\end{array}$ & $\begin{array}{l}1=\text { Oui } \\
0=\text { Non }\end{array}$ & $\begin{array}{l}182(65) \\
98(35)\end{array}$ & + \\
\hline Accès au crédit agricole & CRED & $\begin{array}{l}1=\text { Oui } \\
0=\text { Non }\end{array}$ & $\begin{array}{l}73(26,07) \\
207(73,93)\end{array}$ & + \\
\hline
\end{tabular}

NB : Les valeurs entre parenthèses sont des fréquences relatives

\section{RESULTATS}

\section{Caractéristiques socioéconomiques des maraîchers}

Les résultats obtenus montrent que le maraîchage est conduit par une population à dominance masculine $(92,5 \%)$ sauf des cas rares où la femme représente le chef du ménage $(7,5 \%)$ par suite du décès de son époux ou d'un divorce (Tableau 1). Le maraîchage demeure la principale occupation de la majorité des enquêtés pour lequel ils emblavent en moyenne 2,08 $\pm 1,66$ ha. Ils enregistrent une expérience moyenne d'environ 17 ans dans le maraîchage. Les taux de scolarisation $(32,86 \%)$ et d'alphabétisation $(15,71 \%)$ sont faibles. Les observations sur la taille des ménages de la zone d'étude montrent qu'ils sont pour la plupart de grande taille du fait que les pratiques traditionnelles de communautarisme y sont encore plus fortes. Ces ménages comptent environ 12 personnes en moyenne dont 5 actifs agricoles. Les producteurs enquêtés ont acquis une certaine facilité à se mettre en groupement et de bénéficier de l'appui des agents d'encadrement. Ainsi, 65\% des maraîchers enquêtés appartiennent à des groupements ou associations de maraîchers. En matière de contrôle des ravageurs, l'utilisation de pesticides chimiques constitue la pratique la plus courante ( $91,43 \%$ des enquêtés). Seules $8,57 \%$ des producteurs enquêtés utilisent des biopesticides. Concernant l'encadrement technique des producteurs, 73,93\% parmi eux bénéficient des appuis des agents de l'Agence Territoriale du Développement Agricole (ATDA) et des ONG. L'accès au crédit parait assez problématique pour les producteurs de cultures de contre-saison dans la vallée du Niger. Ainsi, seulement $32,07 \%$ des producteurs enquêtés ont accès au crédit agricole. Ils obtiennent en moyenne auprès des microfinances 177489 FCFA.

Facteurs déterminant l'adoption des pratiques biologique et mécanique et recours à d'autres alternatives aux pesticides

Les résultats du modèle d'estimation des facteurs d'adoption des pratiques biologique et mécanique et recours à d'autres alternatives aux pesticides montrent que le modèle est significatif au seuil de $1 \%$. Le pseudo- $\mathrm{R}^{2}$ de McFadden est de 0,521 (Tableau 2). Les résultats du modèle logistique identifient cinq facteurs déterminants l'adoption des pratiques biologique et 
mécanique et recours à d'autres alternatives aux pesticides. Les variables sexe, superficie emblavée en maraîchage et le nombre d'actifs agricoles sont significatifs au seuil de 5\%; le nombre d'années d'expérience dans le maraîchage et l'éducation formelle sont significatifs au seuil de $10 \%$ (Tableau 2).

Le sexe du maraîcher influence négativement l'adoption des pratiques biologique, mécanique et recours à d'autres alternatives aux pesticides. Ce résultat indique que les femmes adoptent mieux ces pratiques que les hommes.

La superficie emblavée a une influence négative sur la probabilité du choix des pratiques biologique, mécanique et recours à d'autres alternatives aux pesticides. Cependant, il est à relever qu'avec le signe négatif du coefficient, ce résultat nous indique que plus la superficie emblavée est grande, moins le maraîcher aura tendance à adopter cette stratégie face aux ravageurs des cultures.

Le nombre d'actifs agricoles a une influence positive sur l'adoption des pratiques biologique, mécanique et recours à d'autres alternatives aux pesticides. Cela suppose que, plus la main-d'œuvre familiale est disponible dans le ménage de l'enquêté, plus la probabilité qu'il choisisse ces pratiques est élevée.

L'éducation formelle affecte positivement l'adoption des pratiques biologique, mécanique et recours à d'autres alternatives aux pesticides. Ainsi, les maraîchers instruits formellement ont tendance à plus adopter ces pratiques que les maraîchers non instruits.

\section{Facteurs déterminant l'adoption semences paysannes et améliorées} des

L'analyse des résultats du Tableau 3 montre que le modèle d'estimation des facteurs de l'adoption des semences paysannes et améliorées est significatif au seuil de $1 \%$ avec un pseudo- $\mathrm{R}^{2}$ de McFadden de valeur 0,521 . La probabilité d'adoption de ces pratiques est diversement influencée par les variables considérées.

La superficie emblavée pour le maraîchage est significative au seuil de $1 \%$ et influence négativement l'utilisation des semences paysannes et améliorées. Cela suppose que les maraîchers qui emblavent de grande superficie optent moins pour l'utilisation des pratiques culturales.

L'expérience dans le maraîchage a un impact significatif et positif au seuil de $1 \%$ sur l'adoption des semences paysannes et améliorées. Plus le maraîcher est expérimenté, plus il songe à l'utilisation de ces semences. L'âge étant proxy de l'expérience, les jeunes sont donc moins disposés à leur utilisation que les personnes âgées.

L'éducation formelle affecte positivement de manière significative au seuil $1 \%$ l'adoption des semences paysannes et améliorées. Ainsi, les maraîchers instruits ont tendance à plus adopter cette pratique que les maraîchers non instruits.

L'appartenance à un groupement est significative au seuil de $10 \%$ et influence positivement la probabilité que le producteur adopte les semences paysannes et améliorées. Ce résultat traduit que le producteur qui appartient à un groupement opte pour l'utilisation de ces semences.

\section{Déterminants de l'adoption de la pratique d'agroforesterie}

L'analyse du Tableau 4 révèle que le modèle d'estimation des facteurs d'adoption de la pratique d'agroforesterie est significatif au seuil de $1 \%$. Le pseudo- $\mathrm{R}^{2}$ de McFadden est de 0,521 . L'analyse des résultats montrent que les variables ont une influence sur l'utilisation de la pratique d'agroforesterie en matière de gestion de la fertilité des sols de la manière suivante :

L'expérience en maraîchage a un effet significatif et négatif au seuil de 5\% sur l'adoption de l'agroforesterie. Plus le maraîcher est âgé, moins il songe à l'adoption de l'agroforesterie comme stratégie de conservation des sols. Les jeunes sont donc plus disposés à l'utilisation de cette pratique que les personnes âgées.

Le nombre d'actifs agricoles a une influence positive sur l'adoption de l'agroforesterie. Cela suppose que, plus la main-d'œuvre familiale est disponible dans le 
ménage, plus la probabilité le maraîcher adopte l'agroforesterie est élevée.

Le mode d'accès à la terre influence positivement la pratique d'agroforesterie. Ce résultat indique que seuls les maraîchers ayant hérités les terres qu'ils cultivent sont habiletés à planter des arbres sur la parcelle en culture. Les autres, l'ayant acquis par don, seront vus comme s'accaparant de ces terres lorsqu'ils s'adonneront à cette action.

\section{Déterminants de l'adoption des pratiques de conservation des sols et de l'eau}

Le modèle d'estimation des facteurs d'adoption de la pratique de conservation des sols et de l'eau est significatif au seuil de $1 \%$ avec un pseudo- $\mathrm{R}^{2}$ de McFadden égal à 0,408 (Tableau 5). Les résultats $\mathrm{du}$ modèle logistique identifient cinq facteurs déterminants de l'adoption de ces pratiques.

L'expérience en maraîchage est significative au seuil de $10 \%$ et influence négativement l'adoption des pratiques de conservation des eaux et des sols. Cela suppose que les maraîchers expérimentés dans la production des cultures maraîchères adoptent mieux ces pratiques agroécologiques que les moins expérimentés.

La superficie emblavée pour le maraîchage a un effet négatif et significatif au seuil de $10 \%$ sur l'utilisation des pratiques de conservation des eaux et des sols. Cela suppose que les maraîchers qui emblavent de grande superficie optent moins pour l'utilisation de ces pratiques agro-écologiques.

Le nombre d'actifs agricoles a une influence positive et significative au seuil de $5 \%$ sur l'adoption de la conservation des eaux et des sols. Cela suppose que, plus la maind'œuvre familiale est disponible dans le ménage de l'enquêté, plus la probabilité qu'il choisisse cette pratique est élevée.

Le mode d'accès à la terre influence positivement la conservation des eaux et des sols. Les maraîchers ayant hérité les terres prennent des dispositions pour lutter contre l'érosion hydrique et éolienne. Les autres, l'ayant acquis par don, seront vus comme s'accaparant de ces terres lorsqu'ils s'adonneront à cette action.

\section{Déterminants de l'adoption de l'association des cultures et l'élevage}

Le Tableau 6 synthétise les statistiques $\mathrm{du}$ modèle d'estimation de l'adoption de l'intégration de l'élevage dans le maraîchage. Le pseudo- $\mathrm{R}^{2}$ de McFadden est de 0,390. Trois variables expliquent la probabilité d'adoption de l'association des cultures et l'élevage. Il s'agit du sexe du maraîcher, de l'expérience dans le maraîchage et du nombre d'actifs au seuil de $5 \%$.

L'analyse des signes des coefficients liés à ces facteurs révèle que la probabilité d'adoption de l'association de l'élevage augmente avec le nombre d'actifs et lorsque l'enquêté est un homme. Par contre, cette probabilité diminue avec le nombre d'années d'expérience. L'expérience étant proxy de l'âge, on peut déduire de ces résultats que les hommes jeunes ayant à leur disposition de la main d'œuvre adoptent mieux cette pratique que les femmes.

\section{Déterminants de la pratique de rotation et d'association des cultures appropriées \\ Le nombre d'actifs agricoles et} l'éducation formelle sont significatifs au seuil de $5 \%$ et l'expérience au seuil de $10 \%$, sont les facteurs qui déterminent l'adoption de la rotation et d'association des cultures appropriées (Tableau 7).

L'expérience en maraîchage a une incidence positive sur l'adoption de la pratique de rotation ou d'association de cultures appropriées. Plus le producteur est expérimenté, plus il opte pour l'utilisation de cette pratique.

Le nombre d'actifs agricoles influence négativement la pratique de l'association rotation. Plus un ménage a un nombre d'actifs agricoles élevé, moins il est réceptif à l'utilisation de cette pratique. 
Tableau 2: Résultat du modèle d'estimation des facteurs d'adoption des pratiques biologique, mécanique et recours à d'autres alternatives aux pesticides.

\begin{tabular}{|c|c|c|c|}
\hline Variables & Coefficients & Erreurs standard & Signification \\
\hline Sexe & $1,758^{* *}$ & 0,553 & 0,002 \\
\hline $\begin{array}{l}\text { Maraîchage comme activité } \\
\text { principale }\end{array}$ & 0,0978 & 1,714 & 0,954 \\
\hline Expérience en maraîchage & $-0,983 *$ & 0,146 & 0,062 \\
\hline Superficie emblavée & $-0,196^{*}$ & 0,592 & 0,073 \\
\hline Actifs agricoles & $1,407 * *$ & 0,586 & 0,016 \\
\hline Education formelle & $0,915^{* *}$ & 0,617 & 0,031 \\
\hline Education informelle & $-0,528$ & 0,656 & 0,421 \\
\hline Mode d'accès à la terre & 0,297 & 0,577 & 0,606 \\
\hline Accès à la vulgarisation & 0,158 & 0,303 & 0,601 \\
\hline Contacts avec ONG ou projet & 1,973 & 0,636 & 0,152 \\
\hline Appartenance à un groupement & 0,111 & 0,475 & 0,815 \\
\hline Accès au crédit agricole & $-0,383$ & 0,620 & 0,537 \\
\hline \multicolumn{4}{|c|}{ Résumé du modèle } \\
\hline Nombre d'observation & \multicolumn{3}{|c|}{280} \\
\hline Log pseudo likelihood & \multicolumn{3}{|l|}{117,275} \\
\hline Pseudo $\mathrm{R}^{2}$ & \multicolumn{3}{|l|}{$0,521 * * *$} \\
\hline Probabilité de signification & \multicolumn{3}{|l|}{0,000} \\
\hline
\end{tabular}

Tableau 3: Résultat du modèle d'estimation des facteurs de l'adoption des semences paysannes et améliorées.

\begin{tabular}{llll}
\hline Variables & Coefficients & Erreurs standard & Signification \\
\hline Sexe & 0,419 & 0,599 & 0,485 \\
Maraîchage comme activité & 0,929 & 1,885 & 0,622 \\
principale & & & \\
Expérience en maraîchage & $1,824^{* * *}$ & 0,559 & 0,008 \\
Superficie emblavée & $-0,911^{* * *}$ & 0,184 & 0,000 \\
Actifs agricoles & 0,051 & 0,041 & 0,166 \\
Education formelle & $1,383^{* * *}$ & 0,366 & 0,000 \\
Education informelle & 0,00 & 0,026 & 0,992 \\
Mode d'accès à la terre & 0,088 & 0,163 & 0,586 \\
Accès à la vulgarisation & 0,055 & 0,441 & 0,892 \\
Contacts avec ONG ou projet & $-0,02$ & 0,03 & 0,543 \\
Appartenance à un groupement & 0,088 & 0,053 & 0,196 \\
Accès au crédit agricole & $-0,262$ & 0,625 & 0,670 \\
& Résumé du modèle & \\
Nombre d'observation & 280 & & \\
Log pseudo likelihood & 124,389 & & \\
Pseudo R & $0,492^{* * *}$ & & \\
Probabilité de signification & 0,000 & & \\
***significatif à 1\%;**significatif à $5 \%$; *significatif à 10\% &
\end{tabular}


Tableau 4: Résultat du modèle d'estimation des facteurs d'adoption de l'agroforesterie.

\begin{tabular}{|c|c|c|c|}
\hline Variables & Coefficients & Erreurs standard & Signification \\
\hline Sexe & 0,418 & 0,589 & 0,478 \\
\hline $\begin{array}{l}\text { Maraîchage comme activité } \\
\text { principale }\end{array}$ & 0,123 & 0,560 & 0,827 \\
\hline Expérience en maraîchage & $-0,686^{* *}$ & 2,259 & 0,024 \\
\hline Superficie emblavée & $-0,517$ & 0,299 & 0,105 \\
\hline Actifs agricoles & $0,989 * * *$ & 0,320 & 0,002 \\
\hline Education formelle & 0,355 & 0,352 & 0,314 \\
\hline Education informelle & $-0,105$ & 0,703 & 0,882 \\
\hline Mode d'accès à la terre & $0,714 * * *$ & 0,268 & 0,008 \\
\hline Contacts avec ONG ou projet & 0,124 & 0,559 & 0,822 \\
\hline Appartenance à un groupement & $-0,492$ & 0,238 & 0,134 \\
\hline Accès au crédit agricole & $-0,045$ & 0,028 & 0,112 \\
\hline \multicolumn{4}{|c|}{ Résumé du modèle } \\
\hline Nombre d'observation & \multicolumn{3}{|c|}{280} \\
\hline Log pseudo likelihood & \multicolumn{3}{|l|}{128,675} \\
\hline Pseudo $\mathrm{R}^{2}$ & \multicolumn{3}{|l|}{$0,439 * * *$} \\
\hline Probabilité de signification & \multicolumn{3}{|l|}{0,000} \\
\hline
\end{tabular}

Tableau 5: Résultat du modèle d'estimation des facteurs d'adoption des pratiques de conservation des sols et de l'eau.

\begin{tabular}{|c|c|c|c|}
\hline Variables & Coefficients & Erreurs standard & Signification \\
\hline Sexe & $-0,105$ & 0,703 & 0,882 \\
\hline $\begin{array}{l}\text { Maraîchage comme activité } \\
\text { principale }\end{array}$ & 0,094 & 1,712 & 0,949 \\
\hline Expérience en maraîchage & $-0,196 * *$ & 0,592 & 0,073 \\
\hline Superficie emblavée & $-0,090^{*}$ & 0,085 & 0,092 \\
\hline Actifs agricoles & $1,407 * *$ & 0,586 & 0,016 \\
\hline Education formelle & $1,390 * * *$ & 0,364 & 0,000 \\
\hline Education informelle & $-0,527$ & 0,654 & 0,418 \\
\hline Mode d'accès à la terre & $0,622 * * *$ & 0,232 & 0,005 \\
\hline Accès à la vulgarisation & $-0,054$ & 0,075 & 0,470 \\
\hline Contacts avec ONG ou projet & 0,059 & 0,448 & 0,895 \\
\hline Appartenance à un groupement & 0,350 & 0,642 & 0,589 \\
\hline Accès au crédit agricole & $-0,102$ & 0,309 & 0,735 \\
\hline \multicolumn{4}{|l|}{ Résumé du modèle } \\
\hline Nombre d'observation & 280 & & \\
\hline Log pseudo likelihood & 138,49 & & \\
\hline Pseudo $\mathrm{R}^{2}$ & $0,408 * * *$ & & \\
\hline Probabilité de signification & 0,000 & & \\
\hline
\end{tabular}


Tableau 6 : Résultat du modèle d'estimation des facteurs d'adoption de l'association des cultures et l'élevage.

\begin{tabular}{|c|c|c|c|}
\hline Variables & Coefficients & Erreurs standard & Signification \\
\hline Sexe & $1,410^{* *}$ & 0,623 & 0,024 \\
\hline $\begin{array}{l}\text { Maraîchage comme activité } \\
\text { principale }\end{array}$ & $-0,751$ & 0,683 & 0,271 \\
\hline Expérience en maraîchage & $-1,674 * *$ & 0,812 & 0,039 \\
\hline Superficie emblavée & $-0,043$ & 0,035 & 0,213 \\
\hline $\begin{array}{l}\text { Actifs agricoles } \\
\text { Education formelle }\end{array}$ & $\begin{array}{c}1,505^{* *} \\
0,036\end{array}$ & $\begin{array}{l}0,670 \\
0,031\end{array}$ & $\begin{array}{l}0,045 \\
0,237\end{array}$ \\
\hline Education informelle & $-0,924$ & 0,604 & 0,126 \\
\hline Mode d'accès à la terre & 0,841 & 0,805 & 0,296 \\
\hline Accès à la vulgarisation & 0,787 & 0,687 & 0,252 \\
\hline Contacts avec ONG ou projet & 0,351 & 0,599 & 0,558 \\
\hline $\begin{array}{l}\text { Appartenance à un } \\
\text { groupement }\end{array}$ & 1,564 & 1,164 & 0,179 \\
\hline Accès au crédit agricole & $-1,206$ & 2,239 & 0,590 \\
\hline \multicolumn{4}{|l|}{ Résumé du modèle } \\
\hline Nombre d'observation & 280 & & \\
\hline Log pseudo likelihood & 142,025 & & \\
\hline Pseudo $\mathrm{R}^{2}$ & 0,390 & & \\
\hline Probabilité de signification & 0,032 & & \\
\hline
\end{tabular}

Tableau 7: Résultat du modèle d'estimation des facteurs d'adoption de rotation et d'association des cultures.

\begin{tabular}{|c|c|c|c|}
\hline Variables & Coefficients & Erreurs standard & Signification \\
\hline Sexe & $-0,048$ & 0,023 & 0,830 \\
\hline $\begin{array}{l}\text { Maraîchage comme activité } \\
\text { principale }\end{array}$ & $-0,059$ & 0,100 & 0,441 \\
\hline Expérience en maraîchage & $1,572 *$ & 1,062 & 0,095 \\
\hline Superficie emblavée & $-0,062$ & 0,017 & 0,572 \\
\hline Actifs agricoles & $-0,234 *$ & 0,130 & 0,075 \\
\hline Education formelle & 0,921 & 0,479 & 0,148 \\
\hline Education informelle & 0,493 & 0,497 & 0,321 \\
\hline Mode d'accès à la terre & $-0,688$ & 0,477 & 0,170 \\
\hline Accès à la vulgarisation & 0,473 & 0,625 & 0,419 \\
\hline $\begin{array}{l}\text { Contacts avec ONG ou } \\
\text { projet }\end{array}$ & 0,165 & 0,430 & 0,272 \\
\hline $\begin{array}{l}\text { Appartenance à un } \\
\text { groupement }\end{array}$ & 0,470 & 0,535 & 0,377 \\
\hline $\begin{array}{l}\text { Accès au crédit agricole } \\
\text { Résumé du modèle }\end{array}$ & $-0,107$ & 0,708 & 0,890 \\
\hline Nombre d'observation & 280 & & \\
\hline Log pseudo likelihood & 145,025 & & \\
\hline Pseudo $\mathrm{R}^{2}$ & $0,247 * *$ & & \\
\hline Probabilité de signification & 0,047 & & \\
\hline
\end{tabular}




\section{DISCUSSION}

Plusieurs auteurs sont arrivés à la conclusion selon laquelle les facteurs déterminant l'adoption d'une innovation introduite en milieu rural sont liés non seulement à la technologie mais aussi aux conditions socioéconomiques et démographiques du producteur (Yabi et al., 2016). Cependant, cette influence varie d'une technologie à l'autre et selon le contexte. A l'issue des estimations de l'effet des facteurs déterminants l'adoption des pratiques agroécologiques, les résultats montrent que les facteurs socioéconomiques et démographiques ont un effet négatif ou positif sur l'adoption des pratiques considérées.

Comme prédit, le niveau d'instruction des maraîchers contribue énormément à rehausser le taux d'adoption des différentes pratiques agro-écologiques. Ces résultats s'expliqueraient par le fait que l'instruction des maraîchers a un effet sur leur point de vue et leur permet d'appréhender les avantages liés à l'adoption des pratiques agroécologiques sur la santé humaine, l'écosystème ainsi que sur la productivité. Ces résultats corroborent ceux de Adebiyi et al. (2019), Yabi et al. (2016), Roussy et al.(2015) et Ngondjeb et al. (2014) qui avaient trouvé le rôle positif de l'éducation dans l'adoption des pratiques agroécologiques. Par contre, nos résultats sont en contradiction avec ceux de Nambie (2018). Cet auteur avait observé un effet positif du niveau d'instruction sur l'adoption de l'utilisation des extraits botaniques par les maraîchers de la ville de Ouagadougou au Burkina-Faso. De même, Dakin (2008) était arrivé à la conclusion suivant laquelle le niveau d'instruction affectait négativement l'adoption de des technologies vulgarisées par le PDRT.

De l'analyse des résultats, il ressort que le nombre d'années d'expérience est un facteur déterminant de l'adoption des pratiques agro-écologiques en maraîchage dans la zone d'étude. En effet, les maraîchers moins expérimentés sont favorables à l'adoption de la diversification et la rotation des cultures, à l'agroforesterie et la lutte biologique et mécanique et recours à d'autres alternatives aux pesticides et à l'intégration de l'élevage dans le maraîchage. Ces résultats pourraient sous-entendre que du fait de l'avantage comparatif coût bénéfice, les maraîchers expérimentés sont parfois pressés des effets immédiats de ces pratiques agroécologiques, et de ce fait, seraient réticents à s'y investir. Ces résultats viennent ainsi attester en partie ceux de Yabi et al. (2016), de Ouédraogo (2003) et de Zegeye et al. (2001) et qui ont abouti à la conclusion que l'adoption de ces pratiques était influencée par l'expérience des producteurs. De même ces auteurs soulignent que l'adoption de ces technologies exige un certain niveau de risque associé à la décision du choix des innovations. Aussi, soulignent-ils que les jeunes producteurs moins expérimentés sont enclins à prendre plus de risque que les producteurs plus âgés. Mais en ce qui concerne la lutte biologiques contre les ravageurs des cultures, les résultats de l'étude infirment ceux obtenus par Nambie (2018) au Burkina, Hassan (2017) au Kenya et Adetonah et al. (2011) au Bénin et au Ghana. Ils ont rapporté que l'expérience en maraîchage influence positivement et significativement l'utilisation des biopesticides et des extraits aqueux de plantes. Par ailleurs, les maraîchers expérimentés sont favorables à l'utilisation des semences paysannes et améliorées; et à la rotation et l'association des cultures appropriées. Ces résultats se justifieraient par le fait que ces technologies sont des pratiques traditionnelles, mieux maîtrisées par les personnes âgées car l'expérience est proxy de l'âge. Par ailleurs ces derniers seraient très conservateurs et réticents envers les nouvelles technologies.

Les maraîchers propriétaires des terres qu'ils cultivent sont favorables à l'adoption de l'agroforesterie et à la conservation des sols et des eaux. Cette influence positive du statut foncier du maraîcher qu'est le mode direct d'accès à la terre sur l'adoption de l'agroforesterie et sur la conservation des sols 
et des eaux fait penser à la sécurisation foncière. Ainsi, les maraîchers propriétaires des terres qu'ils cultivent seraient disposés à entreprendre des actions permettant de conserver à moyen et à long terme la fertilité des sols. Ce résultat corrobore celui de Diogo et al. (2018) qui ont trouvé que la formalisation des droits de propriété avait une influence positive sur le degré d'investissement des producteurs de maïs et de coton de la commune de Gogounou dans la Gestion Durable des Terres (GDT). C'est ainsi que, Amichi et al. (2016) ont estimé que le statut foncier instable ne sécurise pas les investissements à long terme sur les terres de cultures. Les mêmes auteurs indiquent qu'en interdisant par exemple l'arboriculture, les producteurs pratiquent plusieurs cycles de cultures sans observer de rotation culturale, ou manquent d'apporter les amendements organiques nécessaires au maintien de la fertilité du sol.

La part de la superficie en maraîchage agit en diminuant la probabilité d'adoption des pratiques biologique, mécanique et le recours à d'autres alternatives aux pesticides de même que la conservation des sols et de l'eau et des semences paysannes et améliorées. Ces résultats pourraient s'expliquer par le fait qu'avoir des terres est une chose mais pouvoir les mettre en valeurs avec de bonnes pratiques agricoles en est une autre. Mettre en culture de grandes superficies en semences paysannes et améliorées nécessite des ressources financières plus coûteuses que les semences non certifiées, et des intrants complémentaires tels que les engrais et les insecticides pour le contrôles des ravageurs pour une productivité optimale. Ainsi, l'adoption de ces pratiques agroécologiques nécessiterait assez de temps, de la formation et de travail pour la restauration et la conservation de la fertilité du sol et pour le contrôle et le traitement avec précision des cultures que ne trouveraient pas facilement les exploitations de grandes tailles. Ce résultat met ainsi en évidence que l'adoption d'une technologie de production peut être freinée par le coût supplémentaire de travail alors qu'elle peut être incitée parce qu'elle fait épargner ce coût (McBride et al., 2003).

Plus le nombre d'actifs agricoles est élevé, mieux les producteurs adoptent en grande partie les différents pratiques agroécologiques. Ceci pourrait s'expliquer par le fait que certaines mesures comme les cordons pierreux ou la confection des digues et l'utilisation des extraits aqueux de feuilles de neem ou de papayer seraient couteuses en termes de main d'œuvre. De ce fait, le nombre d'actifs agricoles dont dispose le producteur serait un palliatif des dépenses en main d'œuvre donc, l'encourage à mieux adopter les pratiques agro-écologiques. Ce constat est partagé par Dossa et al. (2018) qui ont estimé que, le nombre croissant d'actifs agricoles permet de répondre au besoin en main d'œuvre qui s'impose au producteur. La décision d'accroître les superficies cultivées diminue la probabilité d'adoption des différentes pratiques agro-écologiques par les maraîchers. Ces résultats s'expliquent certainement par le fait que plus la superficie est élevée plus l'investissement dans les bonnes pratiques cultures culturales augmente. Il convient de souligner que certaines mesures comme les cordons pierreux ou la confection des digues et l'utilisation des extraits aqueux de feuilles de neem ou de papayer seraient couteuses en termes de main d'œuvre.

Le contact avec un agent de vulgarisation ou une ONG ou un projet n'a pas d'effet significatif sur l'adoption des différentes pratiques agro-écologiques en dehors des pratiques biologique, mécanique et recours à d'autres alternatives aux pesticides. Cependant, le signe des coefficients montre que l'effet du contact avec un agent de vulgarisation ou une ONG ou un projet sur l'adoption des différentes pratiques est majoritairement positif mais non significatif. L'impact positif de la vulgarisation ou une ONG ou un projet sur l'adoption des technologies agricoles a été révélé par plusieurs auteurs (Roussy et al., 2015 ; Yabi et al., 2016 ; Issoufou et al., 2017). Les 
maraîchers qui sont en contact avec une de ces structures de vulgarisation, bénéficient d'un encadrement plus rapproché. Ils accèdent donc facilement à l'information et à la formation sur les nouvelles technologies (Issoufou et al., 2017). L'impact non significatif du contact avec un agent de vulgarisation observé sur l'adoption des pratiques agro-écologiques pourrait s'expliquer par une insuffisance de l'appui conseil ou de formation des agents en agriculture agro-écologique.

Les hommes sont plus disposés à adopter les pratiques biologiques, mécanique et recours à d'autres alternatives aux pesticides pour l'entretien des cultures. Cela signifie que les hommes sont plus susceptibles d'allouer plus de superficies au maraîchage agro-écologiques que les femmes. Le fait d'être femme réduit donc la probabilité de l'intensité d'adoption de ces pratiques agro-écologiques dans le maraîchage. Une augmentation des superficies demanderait plus d'entretiens des champs, dévolues aux hommes qui ont plus de force de travail et de disponibilité que les femmes. Ces dernières doivent généralement remplir les fonctions ménagères. Ces résultats sont en partie conformes à ceux de Adekambi et al. (2010) qui ont rapporté que le sexe influence positivement les perceptions des maraîchers sur l'adoption des biopesticides et des extraits aqueux de plantes.

\section{Conclusion}

Les différentes analyses mettent en évidence les déterminants de l'adoption des pratiques agro-écologiques dans la production maraîchère dans la vallée du Niger au Bénin. Ces facteurs varient d'une pratique à une autre. La plupart de ces facteurs sont socioéconomiques et techniques et sont non seulement liée aux producteurs mais aussi à la pratique. L'adoption de des pratiques agroécologiques maintiendraient la fertilité des sols et ainsi amélioreraient le rendement des cultures. Pour cela, il faut non seulement que les maraîchers actuels de l'agroécologie soient organisés, mais qu'ils développent en plus des actions constantes et efficaces de plaidoyer et de lobbying auprès des décideurs techniques et politiques pour une prise en compte effective de leur activité dans les politiques de développement économique et sociale.

\section{CONFLITS D'INTERETS}

Les auteurs de cet article ne signalent aucun conflit d'intérêts.

\section{CONTRIBUTIONS DES AUTEURS}

CCK a collecté, analysé les données, et rédigé l'article. BDT a contribué à l'analyse des données et à la rédaction de l'article. CT et JAY ont supervisé la collecte, l'analyse des données et ont corrigé l'article. Tous les auteurs déclarent avoir lu et approuvé la version finale du manuscrit.

\section{REFERENCES}

Adebiyi KD, Maiga-Yaleu S, Issaka K, Ayena M, Yabi JA. 2019. Déterminants de l'adoption des bonnes pratiques de gestion durable des terres dans un contexte de changement climatique au Nord Bénin : cas de la fumure organique. International Journal of Biological and Chemical Sciences, 13(2): 998-1010. DOI:

https://dx.doi.org/10.4314/ijbcs.v13i2.3.

Adekambi AS, Adegbola YP, Arouna A, 2010. Farmers' perception and agricultural technology adoption. The case of botanical extracts and biopesticides in vegetable production in Benin. Contributed Paper presented at the Joint 3rd African Association of Agricultural Economists (AAAE) and 48th Agricultural Economists Association of South Africa (AEASA) Conference, Cape Town, South Africa, September 19-23, 2010. DOI: http://dx.doi.org/10.22004/ag.econ.9591 7. 
Ahouandjinou MC, Adegbola PY, Yabi JA, Adekambi SA. 2010. Adoption et impact socio-économique de la semimécanisation du procédé de transformation des amandes de karité en beurre au Nord-Bénin. Contributed Paper presented at the Joint 3rd African Association of Agricultural Economists (AAAE) and 48th Agricultural Economists Association of South Africa (AEASA) Conference, Cape Town, South Africa, September 19-23, 2010. $27 \mathrm{p}$.

DOI: http://dx.doi.org/10.22004/ag.econ.9618 6.

Ahouangninou C. 2013. Durabilité de la production maraîchère au sud-Bénin : un essai de l'approche écosystémique. Thèse de Doctorat, Université d'Abomey-Calavi, Bénin, p.333.

Amichi H, Jamin JY, Morardet S, Gharbi I, Azizi A, Faidani F, Ghileb M, Marlet S, Elloumi M. 2016. Le rôle du faire valoir indirect dans le renouvellement générationnel des agriculteurs irrigants en Tunisie. Cahiers Agricultures, 25(3): 1-7.

DOI: http://dx.doi.org/10.1051/cagri/2016022.

Biaou D, Yabi J, Yegbemey R, Biaou G. 2016. Performances technique et économique des pratiques culturales de gestion et de conservation de la fertilité des sols en production maraîchère dans la commune de Malanville, Nord Bénin. International Journal of Innovation and Scientific Research, 21: 2351-8014. http://www.ijisr.issr-journals.org/

CIMMYT, ECONOMICS PROGRAM. 1993. The adoption of agricultural technology: A guide for survey design. Mexico, D.F.: CIMMYT. 88 p.

CIRAD-GRET. 2002. Ministère des Affaires étrangères, Mémento de l'Agronome, 1691p.

Dagnelie P. 2006. Statistique Théorique et Appliquée: 2. Inférence Statistique à 1 et
2 Dimensions (Volume 2, Édition 2). Éditeur De Boeck Supérieur; 736p.

Diogo RVC, Agandan EMM, Nouatin GS, Djedje M. 2017. Modes de gestion de la fertilité des sols des agro-éleveurs peuls au Nord-Ouest du Bénin : implications pour la sécurité alimentaire. Annales de l'Université de Parakou. Sciences Naturelles et Agronomie, 1 : 74-81.

Gravel A. 2016. Les pratiques agroécologiques dans les exploitations agricoles urbaines et périurbaines pour la sécurité alimentaire des villes d'Afrique subsaharienne. Essai présenté au Département de biologie en vue de l'obtention du grade de maître en écologie internationale (M.E.I.).Faculté des sciences, Université de Sherbrooke, Québec, Canada, p.104.

Issoufou OH, Boubacar S, Adam T, Yamba B. 2017. Déterminants de l'adoption et impact des variétés améliorées sur la productivité du mil au Niger. African Crop Science Journal, 25(2): 207 - 220. DOI: http://dx.doi.org/10.4314/acsjv25i2.6.

MAEP (Ministère de l'Agriculture de l'Elevage et de la Pêche), 2017: Cadre programmatique du secteur agricole. Cotonou, Bénin, $154 \mathrm{p}$.

McBride WD., Daberkow SG. (2003).Information and the Adoption of Precision Farming Technologies. Journal of Agribusiness, 21(1): 21-38. DOI:

http://dx.doi.org/10.22004/ag.econ.1467 1.

Ngondjeb Y, Nje P, Havar M. 2011. Déterminants de l'adoption des techniques de lutte contre l'érosion hydrique en zone cotonnière du Cameroun. Revue d'Elevage et de Médecine Vétérinaire des Pays Tropicaux, 64: 9-19. DOI: https://doi.org/10.19182/remvt.10120

Plan Stratégique de développement du Secteur Agricole (PSDSA). 2017. Orientations 
stratégiques 2025, Plan National d'Investissements Agricoles et de Sécurité Alimentaire et Nutritionnelle PNIASAN 2017 - 2021. Version finale, 2017, p.132.

Projet de Soutien à l'Extension de Projet de Soutien à l'Extension de l'Agriculture Agro-Ecologique au Bénin. Etude des pratiques Etude des pratiques agroécologiques au Bénin, 2011. Rapport provisoire, 63p.

Roussy C, Ridier A, Chaib K. 2015. Adoption d'innovations par les agriculteurs : rôle des perceptions et des préférences. Working Paper SMART-LERECO $\mathrm{N}^{\circ} 15-03$.

https://ageconsearch.umn.edu/bitstream/ 206036/2/WP15-03.pdf. Consulté le 25 mars 2019.

Sale A, Folefack PD, Obwoyere GO, Lenah Wati N, Lendzemo WV, Wakponou A. 2014. Changements climatiques et déterminants d'adoption de la fumure organique dans la région semi-aride de Kibwezi au Kenya. International Journal of Biological and Chemical Sciences, 8(2): 680-694. DOI: http://dx.doi.org/10.4314/ijbcs.v8i2.24.
Seye B, Arouna A, Sall SN, Ndiaye AA. 2017. Impact de l'adoption des semences certifiées des variétés améliorées de riz sur le taux de pauvreté: cas du Bénin. Cahiers du Centre Béninois de la Recherche Scientifique et de l'Innovation, 11 : 96 - 103.

Trabelsi M. 2017. Comment mesurer la performance agroécologique d'une exploitation agricole pour l'accompagner dans son processus de transition? Thèse pour l'obtention du grade de Docteur. École Doctorale ED60 Temps, Territoires, Sociétés, Développement Et de l'unité de recherche UMR GRED : Gouvernance, Risque, Environnement, Développement, Université Paul-Valéry Montpellier 3 - France, p.372.

Yabi AJ, Bachabi XF, Labiyi AI, Ode AC, Ayena LR. 2016. Déterminants socioéconomiques de l'adoption des pratiques culturales de gestion de la fertilité des sols utilisées dans la commune de Ouaké au Nord-Ouest du Bénin. International Journal of Biological and Chemical Sciences, 10(2): $779-79 . \quad$ DOI: http://dx.doi.org/10.4314/ijbcs.v10i2.27. 